\title{
Food and Bioprocess Technology
}

\section{Functional properties of plasticized bio-based Poly(lactic acid)_Poly(hydroxybutyrate) (PLA_PHB) films for active food packaging

\author{
--Manuscript Draft--
}

Manuscript Number:

Full Title:

Article Type:

Keywords:

Corresponding Author:

Corresponding Author Secondary

Information:

Corresponding Author's Institution:

Corresponding Author's Secondary Institution:

First Author:

First Author Secondary Information:

Order of Authors:

Abstract:

Funding Information:
FABT-D-16-00947R1

Functional properties of plasticized bio-based Poly(lactic acid)_Poly(hydroxybutyrate)

(PLA_PHB) films for active food packaging

Original Research

bio-films; active packaging; lactic acid oligomers; carvacrol; migration; antibacterial properties

Nuria Burgos, $\mathrm{PhD}$

Universitat d'Alacant

Alicante, Alicante SPAIN

Universitat d'Alacant

Nuria Burgos, PhD

Nuria Burgos, PhD

Ilaria Armentano

Elena Fortunati

Franco Dominici

Francesca Luzi

Stefano Fiori

Francesco Cristofaro

Livia Visai

Alfonso Jiménez

José M Kenny

Order of Authors Secondary Information:

\begin{tabular}{|l|l|}
\hline $\begin{array}{l}\text { Ministerio de Economía y Competitividad } \\
\text { (MAT2014-59242-C2-2-R) }\end{array}$ & Not applicable \\
\hline $\begin{array}{l}\text { Ministerio de Economía y Competitividad } \\
\text { (MAT2014-55778-REDT) }\end{array}$ & Not applicable \\
\hline $\begin{array}{l}\text { Samsung } \\
\text { (GRO PROGRAMME 2012) }\end{array}$ & Not applicable \\
\hline
\end{tabular}

Fully bio-based and biodegradable active films based on poly(lactic acid) (PLA) blended with poly(3-hydroxy butyrate) (PHB) and incorporating lactic acid oligomers (OLA) as plasticizers and carvacrol as active agent were extruded and fully characterized in their functional properties for antimicrobial active packaging. PLA_PHB films showed good barrier to water vapor, while the resistance to oxygen diffusion decreased with the addition of OLA and carvacrol. Their overall migration in aqueous food simulant was determined and no significant changes were observed by the addition of carvacrol and OLA to the PLA_PHB formulations. However, the effect of both additives in fatty food simulant can be considered a positive feature for the potential protection of foodstuff with high fat content. Moreover, the antioxidant and antimicrobial activities of the proposed formulations increased by the presence of 
carvacrol, with enhanced activity against S.aureus if compared to E.coli at short and long incubation times. These results underlined the specific antimicrobial properties of these bio-films suggesting their applicability in active food packaging. 


\section{Functional properties of plasticized bio-based Poly(lactic}

\section{acid)_Poly(hydroxybutyrate) (PLA_PHB) films for active food packaging}

\section{Dear Editor,}

Thank you and the referees for your very accurate and useful comments to improve our manuscript. Please find below our answer to the referee's comments.

Our answers in red. We have also highlighted in red the changes introduced in the manuscript.

\section{Reviewer \#1 (Comments to the Author (Required)):}

The paper "Functional properties of plasticized bio-based Poly(lactic acid)_Poly(hydroxybutyrate) (PLA_PHB) films for active food packaging" deals with the characterization of a bio-based blend and active film. In particular the authors studied the effect of lactic acid oligomers and carvacrol on the oxygen and water vapour barrier properties, the antioxidant and antimicrobial film capacity, and their overall migration. Although the manuscript is well written and results are clearly reported, I suggest major revision.

As general comment I disagree with the objective indicated by the authors in the introduction (line 16). If the objective of the work was optimize the formulation of film, a different experimental design should have been used. The real objective of the work is to evaluate the effect of plasticizer and carvacrol on the functional properties of the film. To get this aim the authors choose to study two concentration on lactic acid oligomers and one concentration of carvacrol. Which experimental design did you choose? Why did you analyze the results by using one way ANOVA? The independent factors are two and along the test the authors reports a interaction between the two independent parameters (page 8 line 22: synergetic plasticizing effect) that has not been studied by statistical analysis. I suggest the authors to consider a defined experimental design (ex. Full factorial design) 
and to analyze the results in accordance with the experimental design chosen.

We thank the reviewer for his/her valuable comments to improve our manuscript.

We decided to evaluate all possibilities regarding the use of two different concentrations of

lactic acid oligomers and just one concentration of carvacrol with just one-way ANOVA, since strictly speaking just one independent variable was considered. Nevertheless, all references to synergetic effects have been deleted in the manuscript since it is not correctly expressed in the document, and no comments on that way should be introduced. We have deleted/changed the wording in page 1 line 43, page 8 lines 22 and 59, page 9 line 51, page 10 line 61 and page 12 line 12 of the previous manuscript to avoid misunderstanding in this subject on the study of the effects in properties of the OLA concentration.

In addition, the objective of this study was clarified in the last paragraph of the Introduction section (Page 3) as follows:

"The aim of this study was the evaluation of some of the main functional properties of innovative PLA_PHB films to assess their capabilities to be used in food packaging. Barrier properties, antioxidant and antibacterial performance and disintegration behavior have been evaluated. Finally, the overall migration of the main components into selected food simulants has been also determined".

\section{Details comments:}

Page 4 line 2: why did you decide to work with a film of $250 \mathrm{~mm}$ ? isn't it too thick?

Our OTR equipment limits the diameter of the films at 140 x $140 \mathrm{~mm}^{2}$. Therefore, it was necessary to process extruded samples by compression molding in order to obtain circular films with the adequate dimensions, and thicknesses between 200 and $250 \mu \mathrm{m}$ to avoid formation of micro-holes in the films that could result in failure in barrier testing. These films were only used for the evaluation of the gas barrier properties. The rest of the analysis (overall migration, disintegration and antioxidant and antimicrobial tests) were performed by using films with thicknesses between 20 and $60 \mu \mathrm{m}$ and $40 \mathrm{~mm}$ of width, obtained by 
extrusion with the adequate nozzle, being these dimensions more realistic to the real situation in films for food packaging.

Some additional information about the film processing parameters has been included in the Materials and Method Section in order to clarify this point (Page 4):

"It was necessary to process extruded samples by compression molding by using a Hot Press Carver Inc. (Wabash, Indiana, USA) in order to obtain circular films with the adequate dimensions for the evaluation of the gas barrier properties (14 $\mathrm{mm}$ of diameter). Materials were melted at $170{ }^{\circ} \mathrm{C}$ between the plates for $5 \mathrm{~min}$ at atmospheric pressure, and then it was gradually increased up to $5 \mathrm{MPa}$ for 2 min and kept for 5 extra min. A cooling step to room temperature was performed at atmospheric pressure and films with thicknesses between 200 and $250 \mu \mathrm{m}$ were obtained in order to avoid the formation of micro-holes that could result in failure in barrier testing".

Page 12 line 20: I disagree with this conclusion. The antimicrobial capacity of the film is not the best one could expect. Moreover, the fact that the antimicrobial activity against both strains was higher at short incubation times is not a positive results because it means that the release in too fast. Please add discussion on it.

According to the reviewer's suggestion, a brief discussion has been included in the revised manuscript (Page 12):

"Moreover, the antimicrobial activity against both strains of PLA_PHB films was significantly enhanced by the presence of both $10 \mathrm{wt} \%$ of carvacrol and OLA (15 or 20 $\mathrm{wt} \%$ ), showing a bactericidal effect just after $3 \mathrm{~h}$ of incubation that is maintained after $24 \mathrm{~h}$ (Fig. 2A and Fig 2B)"'.

Therefore, the following sentences were removed in order to clarify this point: "Interestingly, the antimicrobial activity against both strains was higher at short incubation times (Fig. 2A and Fig 2B)". (Page 12 line 1 in the previous manuscript) and: "Therefore, it could be concluded that the incorporation of $10 \mathrm{wt} \%$ of carvacrol to PLA_PHB films 
plasticized with 15 or $20 \mathrm{wt} \%$ OLA showed their potential as antimicrobial packaging material". (Page 12 line 20 in the previous manuscript)

Reviewer \#2: Comments on the FABT-D-16-00947 manuscript The subject of this manuscript is actual and important for the food industries and this research group is developing a continuous and coherent work in this area. This manuscript describe more one result of the functional properties of plasticized bio-based poly (lactic acid) - poly (hydroxybutyrate) films active packaging. This manuscript is well written, the experimental methodology is similar to one already presented in earlier works of this group with modifications suitable to attend this manuscript objectives. Results are well presented discussed consistently. Therefore I recommend this manuscript to be published in the FABT journal.

We thank the reviewer by his/her positive comments to improve our manuscript.

Two suggestions are presented but they are not required for this manuscript publication: 1 - A briefly description of the blend processing could improve the paper reading without searching it in the earlier works (details of this would be found in the references already given).

More details on blend processing conditions have been introduced in the Materials and Methods Section in order to improve our manuscript, in agreement with the reviewer's suggestion (Page 3-4).

2 - Authors may comment why they have not included the PLA_15PHB_30OLA-10Carv blend in this study. Note that Armentano et al. (2015b) have concluded that PLA_PHB blend with $30 \mathrm{wt}$ \% OLA was the optimum formulation for food packaging (in comparison to PLA_PHB blends with 15 and 20 wt. \% OLA).

In our previous paper (Armentano et al. (2015b)), binary and ternary formulations based on PLA_PHB and different amounts of OLA were studied. The formulation of PLA_PHB_30OLA offered the best compromise between ductile and gas barrier properties 
with no migration problems. However, in the present study, the quaternary systems based on OLA and carvacrol were processed with a lower content of OLA (20 and 15 wt \%) since it was expectable that $10 \mathrm{wt} \%$ of carvacrol could induce additional plasticizing effects.

Furthermore, from our previous experience, the combination of $30 \mathrm{wt} \%$ of OLA and 10 wt\% Carvacrol could induce a negative mechanical behavior. This is the reason why we decided to avoid the additive effects of carvacrol and OLA in plasticizing the PLA_PHB matrix by reducing the OLA content to $15-20 \mathrm{wt} \%$ which could be more realistic in consideration of the intended application of these films.

A new sentence was introduced in the Materials and Methods Section in order to clarify this point, according to the reviewer's suggestion (Page 3-4):

"The highest OLA content used there was not included in this study because, from our previous experience, its combination with $10 \mathrm{wt} \%$ carvacrol could induce additional plasticizing effect with exudation and problems during processing.". 


\title{
Functional properties of plasticized bio-based Poly(lactic acid)_Poly(hydroxybutyrate) (PLA_PHB) films for active food packaging
}

\author{
Nuria Burgos $^{1 *}$, Ilaria Armentano ${ }^{2}$, Elena Fortunati ${ }^{2}$, Franco Dominici ${ }^{2}$, Francesca Luzi ${ }^{2}$, Stefano Fiori ${ }^{3}$, \\ Francesco Cristofaro ${ }^{4}$, Livia Visai ${ }^{4,5}$, Alfonso Jiménez ${ }^{1}$, José M. Kenny ${ }^{2}$ \\ ${ }^{1}$ University of Alicante, Dpt. Analytical Chemistry, Nutrition \& Food Sciences, 03690 San Vicente del Raspeig (Spain) \\ ${ }^{2}$ University of Perugia, Civil and Environmental Engineering Department, UdR INSTM, Strada di Pentima 4, 05100 Terni (Italy) \\ ${ }^{3}$ Condensia Química, S.A. C/Junqueras, 16-11A, 08003, Barcelona, Spain \\ ${ }^{4}$ Molecular Medicine Department, Center for Health Technologies (CHT), UdR INSTM, University of Pavia, Viale Taramelli 3/B, \\ 27100 Pavia (Italy) \\ ${ }^{5}$ Department of Occupational Medicine, Toxicology and Environmental Risk, S. Maugeri Foundation, IRCCS, Via S. Boezio, 28 - \\ 27100 Pavia (Italy) \\ *Corresponding author: nuria.burgos@ua.es (N. Burgos). Phone: +34 965903400, ext.1187.
}

\begin{abstract}
Fully bio-based and biodegradable active films based on poly(lactic acid) (PLA) blended with poly(3-hydroxy butyrate) (PHB) and incorporating lactic acid oligomers (OLA) as plasticizers and carvacrol as active agent were extruded and fully characterized in their functional properties for antimicrobial active packaging. PLA_PHB films showed good barrier to water vapor, while the resistance to oxygen diffusion decreased with the addition of OLA and carvacrol. Their overall migration in aqueous food simulant was determined and no significant changes were observed by the addition of carvacrol and OLA to the PLA_PHB formulations. However, the effect of both additives in fatty food simulant can be considered a positive feature for the potential protection of foodstuff with high fat content. Moreover, the antioxidant and antimicrobial activities of the proposed formulations increased by the presence of carvacrol, with enhanced activity against S.aureus if compared to E.coli at short and long incubation times. These results underlined the specific antimicrobial properties of these bio-films suggesting their applicability in active food packaging.
\end{abstract}

Keywords: bio-films; active packaging; lactic acid oligomers; carvacrol; migration; antibacterial properties. 


\section{Introduction}

Food packaging systems are designed to protect food from environmental influences, such as microbial or chemical degradation, oxidation, light radiation or moisture. In this context, the extension of shelf-life and the reduction of the environmental impacts to the packaged food have attracted the researcher's interest, orienting their activities to develop innovative solutions in line of the raising consumer's requests and the conservation of food products (Alboofetileh et al. 2014; Coma 2008).

The use of polymer-based packaging systems shows many advantages, since they are more flexible, reducing weight and energy requirements for their production. In particular, biopolymers, such as polylactic acid (PLA) and/or poly(3hydroxybutyrate) (PHB) show important advantages to fight against the environmental problems produced by plastic waste (Armentano et al. 2015b; Zhang and Thomas 2011). PLA shows properties comparable to polystyrene and poly(ethylene terephthalate), with good biodegradation abilities (Chaiwutthinan et al. 2015) and biocompatibility (De Silva et al. 2015). It is classified as GRAS (Generally Recognised As Safe) and approved by the US Food and Drug Administration (FDA) for contact with food (Hwang et al. 2012).

However, practical applications of PLA are often limited by its inherently brittle nature. Blending with rubber particles, which serve as stress concentrators and allow for ductile behavior, has been accomplished with a variety of elastomers such as poly(E-caprolactone), low-density polyethylene, and polyisoprene (Delgado and Hillmyer 2014; Armentano et al. 2015b). The modification of PLA by blending with an aliphatic polyester, such as PHB, with high crystallinity and melting point has been reported to improve the physical, mechanical and barrier properties of pure PLA, providing valuable materials for packaging (Armentano et al. 2015a; Arrieta et al. 2014a). PHB and PLA could be processed together due to their similar melting temperatures and PHB can also act as nucleating agent for PLA (Zhang and Thomas 2011).

Other strategy to improve PLA ductile properties is by plasticization. Oligomer lactic acid (OLA) is a bio-based plasticizer to increase PLA ductility with a significant reduction in the polymer glass transition temperature $\left(\mathrm{T}_{\mathrm{g}}\right)$. The introduction of OLA in PLA matrices resulted in highly homogeneous and stable films (Burgos et al. 2014; Armentano et al. 2015a; Burgos et al. 2013).

The possibility to add specific bioactive additives to biopolymers allows us to modulate their functional properties, while maintaining their inherent biodegradability and presenting potential to control bacterial growth in food products. Inhibition is possible with specifically targeted release mechanisms of the active compounds to allow the migration of encapsulated bioactive agents from the film matrix to the package headspace or onto the food surface at a controlled rate (Boumail et al. 2013; Coma 2008). This is a new generation of active materials used to improve the quality and 
safety of food products during storage (Salmieri et al. 2014; Sanchez-Garcia et al. 2008; Ramos et al. 2012; Ramos et al. 2014a).

Food poisoning caused by E.coli and other food spoilage microorganisms is a problem to be solved by the addition of preservatives, but consumers concerns on the use of synthetic additives are growing. Antibacterials extracted from natural sources, such as essential oils (EO), show the desired antimicrobial activity with no harmful effect to food. Among them, oregano EO is one of the most effective in their antimicrobial performance. Carvacrol and thymol, the major components of oregano EO, are legally registered flavouring substances and their antimicrobial properties have been reported (Lambert et al. 2001; Guarda et al. 2011).

The aim of this study was the evaluation of some of the main functional properties of innovative PLA_PHB films to assess their capabilities to be used in food packaging. Barrier properties, antioxidant and antibacterial performance and disintegration behavior have been evaluated. Finally, the overall migration of the main components into selected food simulants has been also determined.

\section{Materials and methods}

\section{Materials}

Poly(lactic acid) commercial grade (96 \% L-LA) (PLA 3051D) (specific gravity $=1.25 \mathrm{~g} \mathrm{~mL}^{-1}$, number molar mass, Mn

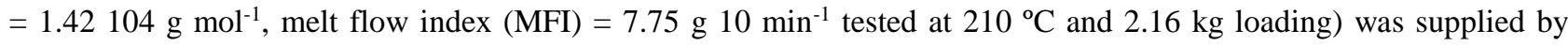
NatureWorks ${ }^{\circledR}$ Co. LLC (Blair, NE, USA). Poly(hydroxybutyrate) $(\mathrm{PHB})\left(\right.$ density $=1.25 \mathrm{~g} \mathrm{~mL}^{-1}, \mathrm{MFI}=15-30 \mathrm{~g} 10$ $\min ^{-1}$ tested at $190{ }^{\circ} \mathrm{C}$ and $2.16 \mathrm{~kg}$ loading) was purchased from NaturePlast (Caen, France). Carvacrol (> $\left.98 \%\right)$ was supplied by Sigma-Aldrich (Madrid, Spain) and it was selected as antimicrobial and antioxidant active additive. An oligomer of lactic acid (OLA) (slightly colored liquid) provided by Condensia Química S.A. (Barcelona, Spain) was selected as the most adequate bio-based plasticizer. OLA was synthesised by following a licensed method (Fiori and Ara 2009), with $\mathrm{Mn}=957 \mathrm{~g} \mathrm{~mol}^{-1}$ (determined by size exclusion chromatography) and glass transition temperature around $-37^{\circ} \mathrm{C}$ (determined by differential scanning calorimetry, DSC).

\section{Processing}

Active films were obtained by mixing PLA with 15 wt $\%$ PHB and the selected additives (OLA and carvacrol), as reported elsewhere (Armentano et al. 2015a). PLA and PHB pellets were dried to avoid the undesirable hydrolysis during processing, while OLA was pre-heated at $100{ }^{\circ} \mathrm{C}$ for 5 min to ensure the liquid state during extrusion.

Two different OLA concentrations (15 and $20 \mathrm{wt} \%$ ) were used, since they were the most adequate as reported in a previous study (Burgos et al. 2013), while the carvacrol content was selected at 10 wt\% (Ramos et al. 2014a). The highest OLA content used there was not included in this study because, from our previous experience, its combination 
with 10 wt \% carvacrol could induce additional plasticizing effect with exudation and problems during processing. The

different formulations used in this study are shown in Table 1. Blends were processed in a twin screw microextruder (Dsm Explore 5\&15 CC Micro Compounder) and films with $40 \mathrm{~mm}$ of width and thickness between 20 and $60 \mu \mathrm{m}$ were obtained by using the adequate nozzle. The temperature profile was set up at $180-190-200{ }^{\circ} \mathrm{C}$ in the three extruder heating zones and $100 \mathrm{rpm}$ of screw speed was used. PLA and PLA_15PHB blends were mixed for 6 min, while for the ternary and quaternary systems both polymers were previously mixed for 3 min (with the incorporation of OLA in the quaternary blends) and carvacrol was added immediately for 3 min of additional mixing.

It was necessary to process extruded samples by compression molding by using a Hot Press Carver Inc. (Wabash, Indiana, USA) in order to obtain circular films with the adequate dimensions for the evaluation of the oxygen barrier properties (14 mm of diameter). Materials were melted at $170{ }^{\circ} \mathrm{C}$, maintained between the plates for 5 min at atmospheric pressure, and then it was gradually increased up to $5 \mathrm{MPa}$ for 2 min and kept for 5 extra min. A cooling step to room temperature was performed at atmospheric pressure and films with thicknesses between 200 and $250 \mu \mathrm{m}$ were obtained in order to avoid the formation of micro-holes that could result in failure in barrier testing.

\section{Table 1}

\section{Functional Characterization}

The suitability of the proposed formulations as active systems for food packaging was evaluated by testing the oxygen and water vapor barrier properties, the overall migration into selected food simulants, the antioxidant activity and the antimicrobial effect against two bacteria commonly present in food, i.e. Escherichia coli (Gram-negative) and Staphylococcus aureus (Gram-positive). In addition, the disintegrability under composting conditions of the different films formulated in this study was evaluated. Experimental details of all these tests are discussed below.

\section{Barrier Properties}

The oxygen transmission rate (OTR) tests were performed in an Oxygen Permeation Analyser (Model 8500), from Systech Instruments (Metrotec S.A, Spain). Films with homogeneous thickness were clamped in the diffusion chamber at $23 \pm 1{ }^{\circ} \mathrm{C}$ and pure oxygen $(\geq 99.9 \%)$ was injected at 2.5 bar. The oxygen volumetric flow rate per unit area of the film and time unit (OTR, $\mathrm{cm}^{3} \mathrm{~m}^{-2} \mathrm{day}^{-1}$ ) was continuously monitored until the steady state was reached. The permeability coefficient is dependent on the film thickness and it is proportional to OTR*e (e $=$ thickness, mm). Conversely, tests were performed in triplicate and were expressed as OTR*e in order to compare the oxygen barrier properties of all the studied films. Film average thickness $( \pm 0.001 \mathrm{~mm})$ was measured at room temperature by using a Digimatic Micrometer Series 293 MDC-Lite (Mitutoyo, Japan) from ten random positions. 
Water vapor permeability (WVP, $\mathrm{kg} \mathrm{m} \mathrm{Pa}^{-1} \mathrm{~s}^{-1} \mathrm{~m}^{-2}$ ) was determined by applying the desiccant method included in the

ASTM E96/E96 M-05 standard (ASTM 2005), and it was calculated by using Eq. (1).

$W V P=\frac{W V T \times e}{\Delta P}$

Where WVT $\left(\mathrm{kg} \mathrm{s}^{-1} \mathrm{~m}^{-2}\right)$ is the water vapor transmission rate, $e(\mathrm{~m})$ is the average film thickness and $\Delta \mathrm{P}(\mathrm{Pa})$ is the vapor pressure difference between both sides of the films, calculated by using Eq. (2).

$\Delta P=S\left(R_{1}-R_{2}\right)$

$S(\mathrm{~Pa})$ is the saturation vapor pressure at the testing temperature and $\mathrm{R}_{1}, \mathrm{R}_{2}$ are the relative humidities in the climate chamber and inside the dish, respectively. In the WVP tests, samples of $90 \mathrm{~mm}$ diameter were sealed with paraffin to the testing stainless steel dishes containing anhydrous calcium chloride (pre-dried at $200{ }^{\circ} \mathrm{C}$ for $2 \mathrm{~h}$ ) as desiccant agent. These dishes were placed in a climate chamber Dycometal-CM81 (Barcelona, Spain) at controlled conditions $\left(23 \pm 1{ }^{\circ} \mathrm{C}\right.$ and $50 \pm 2 \% \mathrm{RH})$ and were weighed periodically until the steady state was reached. The weight change compared with the initial mass, $G( \pm 0.1 \mathrm{mg})$, was plotted versus time at $24 \mathrm{~h}$ intervals, $t(\mathrm{~h})$. Linear regression was used to calculate the slope of a fitted straight line $(G / t)$ that allows the WVT determination by following Eq. (3).

$W V T=\frac{G / t}{A}$

Where $\mathrm{A}$ is the effective area of the tested films $\left(0.01 \mathrm{~m}^{2}\right)$. All WVP values reported in this work are the average of three replicates tested for each sample $(n=3) \pm$ standard deviation $(\mathrm{SD})$.

\section{Overall Migration Tests}

The overall migration tests for all films were performed by using ethanol $10 \%$ (v/v) as aqueous food simulant (simulant A) (EC 2011) and isooctane as the alternative fatty food simulant (Commission Directive 2002/72/EC) (EC 2002). Rectangular film sheets $\left(2.5 \times 10.0 \mathrm{~cm}^{2} \times 0.06 \mathrm{~mm}\right)$ were immersed into $25 \mathrm{~mL}$ of each food simulant and were kept in an oven at $40{ }^{\circ} \mathrm{C}$ for 10 days in ethanol $10 \%(\mathrm{v} / \mathrm{v})$ or in the climate chamber at $20{ }^{\circ} \mathrm{C}$ for 2 days in isooctane. These test conditions were the most restrictive between those indicated in the current legislation for materials intended to be in contact with food at room temperature or below for more than $24 \mathrm{~h}$. Once the maximum contact time was reached, films were removed and simulants were evaporated and dried at $105^{\circ} \mathrm{C}$ for $30 \mathrm{~min}$ in an oven. The non-volatile residue was determined by using an analytical balance $( \pm 0.1 \mathrm{mg})$ until constant weight $( \pm 0.5 \mathrm{mg})$. The overall migration values are expressed in $\mathrm{mg} \mathrm{kg}^{-1}$ of simulant as the average of three replicates \pm standard deviation (SD).

\section{Antioxidant activity}

The antioxidant performance of PLA_15PHB materials containing carvacrol was determined by using the DPPH method (Byun et al. 2010; Ramos et al. 2014a). This method is based on the radical scavenging ability of samples against the stable free radical DPPH (2,2-diphenyl-1-picrylhydrazyl), which has a natural purple color in solution but, 
upon reduction by the antioxidant compound, the absorption at $517 \mathrm{~nm}$ decreases and becomes colorless (Ramos et al.

2014a; Wu et al. 2012). Rectangular sheets of films $(0.05 \pm 0.01 \mathrm{~g})$ (per triplicate) were immersed in $10 \mathrm{~mL}$ of pure methanol and kept at $40^{\circ} \mathrm{C}$ for $24 \mathrm{~h}$ in an oven, as previously reported for the determination of the carvacrol content in PLA_PHB films (Armentano et al. 2015a). A portion of the extract $(0.5 \mathrm{~mL})$ was mixed with $2 \mathrm{~mL}$ of a DPPH solution in methanol $(0.06 \mathrm{mM})$ and this solution was shaken vigorously at room temperature. The absorbance of the solution was measured every 30 seconds until stabilization by using a Biomate-3 UV-VIS Spectrophotometer (Thermospectronic, AL, USA). The ability of each solution to scavenge the stable radical DPPH was calculated as the inhibition percentage (I \%) according to Eq. 4.

$I(\%)=\frac{A_{\text {control }}-A_{\text {sample }}}{A_{\text {control }}} \times 100$

where $A_{\text {control }}$ is the absorbance of the control solution at $\mathrm{t}=0 \mathrm{~min}$ (using methanol instead of the sample) and $A_{\text {sample }}$ is the absorbance of the tested extract after incubation in the dark for $210 \mathrm{~min}$. The values reported here are the average of three replicates \pm standard deviation $(\mathrm{SD})$.

\section{Antimicrobial activity}

The determination of the in vitro susceptibility of bacteria to active agents permits the analysis of the potential of films to protect food against spoilage bacteria. The antimicrobial effect of PLA_PHB and PLA_PHB_OLA films containing carvacrol was evaluated by the agar disk diffusion method and by the CFU/mL method after direct contact with bacterial suspensions. Films without carvacrol were also tested and used as controls. The agar disk diffusion method was used to reproduce the situation when films are in direct contact with food with release of the antimicrobial agent to protect food against the microbial proliferation. This method is recommended by the Clinical and Laboratory Standards Institute (CLSI 2015) for materials intended to be in contact with food. Bacterial cultures (E. coli, CECT 434 and S. aureus, CECT 239) were supplied by the Spanish Type Culture Collection (CECT, University of Valencia, Spain). Isolated colonies of each microorganism, obtained from their individual incubation on nutrient agar plates at $37^{\circ} \mathrm{C}$ for $24 \mathrm{~h}$, were growth in Mueller Hinton Broth at the same temperature for $18 \mathrm{~h}$ in order to standardize their density prior to the test ( 0.5 in the McFarland scale). Two successive (1:10) dilutions in buffered peptone water were prepared to get the

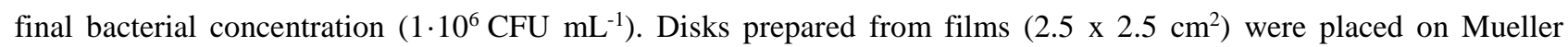
Hinton Agar plates, previously spread with $0.1 \mathrm{~mL}$ of the inoculum suspension, and they were incubated at $37^{\circ} \mathrm{C}$ for 24 h. Therefore, the antimicrobial potential of each film was evaluated by observing the inhibition zones for the bacteria growth. All tests were performed in duplicate for each formulation.

The CFU/mL assay was also carried out in E.coli strain grown in Luria Bertani Broth (LB) (Difco, Detroit, MI, USA) and S. aureus in Brian Heart Infusion (BHI) (Difco) maintained overnight under aerobic conditions at $37^{\circ} \mathrm{C}$ using a 
shaker incubator (New Brunswick Scientific Co., Edison, NJ, USA). These cultures were reduced to a final density of $1 \cdot 10^{10}$ cells $\mathrm{mL}^{-1}$ as determined by comparing the $\mathrm{OD}_{600}$ of the sample with a standard curve relating $\mathrm{OD}_{600}$ to the cell number. The antimicrobial activity of PLA_PHB plasticized blend films with/without carvacrol was evaluated in 100 $\mu \mathrm{L}\left(1 \cdot 10^{4} \mathrm{CFU} \mathrm{mL} \mathrm{mL}^{-1}\right)$ of an overnight diluted cell suspension of $E$. coli or $S$. aureus. They were added to each sample seeded at the bottom of a 96-well tissue culture plate and incubated at $37{ }^{\circ} \mathrm{C}$ for $3 \mathrm{~h}$ and $24 \mathrm{~h}$, respectively. At the end of each incubation time, the bacterial suspensions were serially diluted and plated on the LB (E. coli) or BHI (S. aureus) agar plates and incubated for $24 \mathrm{~h} / 48 \mathrm{~h}$ at $37{ }^{\circ} \mathrm{C}$. Cell survival was expressed for each bacterial strain as the ratio between the bacteria CFU surviving on PLA_PHB plasticized films with/without carvacrol and bacteria CFU grown on neat PLA films. S. aureus and E. coli cells grown on PLA were used as positive controls. The bacterial viability in positive control experiments was used as the reference $(100 \%)$. Levels of bacteria surviving fractions on PLA_PHB plasticized films with/without carvacrol were expressed as the percentage of surviving fractions of both bacterial strains compared with the positive control. All data are expressed as the average of three replicates \pm standard deviation (SD).

\section{Disintegrability in composting conditions}

The disintegrability under composting conditions of PLA, PLA_PHB, and PLA_PHB_OLA_Carv systems was assessed by following the ISO-20200 standard test (ISO 2004), that simulates a real composting process. Specifically, more than $90 \%$ of the initial sample weight should not be retained in a $2 \mathrm{~mm}$ sieve after 90 days. The organic substrate was prepared by mixing compost (supplied by Gesenu S.p.A.) with a synthetic biowaste prepared with starch, sugar, sawdust, rabbit food, oil and urea. In addition, the water content of the substrate should be maintained at around 50 wt $\%$, while the aerobic conditions in all areas of the composting test container were guaranteed by mixing it carefully. Injection molded probes $\left(15 \times 5 \times 2 \mathrm{~mm}^{3}\right)$ were buried at 4-6 $\mathrm{cm}$ depth in perforated boxes containing the prepared substrate and were incubated at $58{ }^{\circ} \mathrm{C}$ (Hakkarainen et al. 2000; Kunioka et al. 2006). Samples were recovered at different disintegration times, washed with distilled water and dried in an oven at $37{ }^{\circ} \mathrm{C}$ for $24 \mathrm{~h}$ to obtain the final weight. The disintegrability data was obtained by normalizing the weight of samples to the initial ones, at different stages of incubation. Photographs of all samples after test were taken for visual observation and comparison.

\section{Statistical analysis}

One way analysis of variance (ANOVA) was carried out on results obtained for barrier properties, migration and antioxidant performance. The statistical program Statgraphics Centurion 16.1.18 (StatPoint, Inc., Warrenton, USA) was used for such purpose. Tukey's multiple sample comparison test with $95 \%$ confidence level $(\mathrm{P}<0.05)$ was used to identify significant differences between data. Additionally, differences in the antimicrobial study under different experimental conditions were calculated using one-way analysis of variance (ANOVA), followed by Bonferroni's post 
hoc test. A two-tailed $\mathrm{P}$ value $<0.05$ was considered statistically significant. These calculations were generated using GraphPad Prism 5.0 (GraphPad Inc., San Diego, CA).

\section{Results and Discussion}

\section{Barrier Properties}

The values for the oxygen transmission rate per film thickness (OTR*e) and the water vapor permeability obtained for all PLA and PLA_PHB based films are reported in Table 2. The addition of $15 \mathrm{wt} \%$ of PHB to the PLA matrix resulted in a significant $(\mathrm{P}<0.05)$ improvement in the oxygen barrier properties, as the consequence of the intrinsic high crystallinity of PHB to induce the formation of a crystalline order in the PLA matrix, as already reported in previous works (Armentano et al. 2015a, 2015b). However, the addition of carvacrol to the blend induced a significant $(\mathrm{P}<0.05)$ increase in OTR*e values, which were up to ca. $39 \%$. This behavior can be explained by considering the increase in the free volume and chain mobility in the PLA_PHB blend caused by the plasticizing effect induced by carvacrol, in agreement with results previously reported in the thermal characterization of these materials (Armentano et al. 2015a). Moreover, other authors reported similar effects on the oxygen permeability in other PLA-based blends for carvacrol (Byun et al. 2010; Ramos et al. 2014b; Jamshidian et al. 2012), D-limonene (Arrieta et al. 2014a) and different plasticizers (Arrieta et al. 2014b).

\section{Table 2}

The reduction in the resistance of these films to the oxygen diffusion was even higher after the addition of OLA. In particular, the incorporation of OLA at both concentrations used in this work (15 and $20 \mathrm{wt} \%)$ increased OTR*e values in around 200 and $270 \%$, respectively. In a previous work, the expected plasticizing effect of OLA in PLA_PHB systems was reported as the main cause of the reduction in the oxygen barrier (around $70 \%$ of reduction with the addition of $20 \mathrm{wt} \%$ OLA) and it was associated to the increase in the free volume available to the difussion of oxygen molecules through the material's structure as well as the higher mobility of the macromolecular chains induced by the presence of the plasticizer, as previously reported after the DSC tests performed to these blends (Armentano et al. 2015b). The higher value for OTR*e observed in the quaternary films is an interesting feature, since it suggests that the selected additives, OLA and carvacrol, showed plasticizing effect, resulting in the higher mobility of the macromolecular chains and the increase in oxygen difussion rate, in agreement with results obtained by DSC in these multifunctional systems (Armentano et al. 2015a). In summary, the improvement in the PLA barrier properties to oxygen caused by the presence of the semi-crystalline PHB is counteracted by the strong plasticizing effect produced by the presence of OLA and carvacrol. However, OTR*e values obtained for these films are in the same order to those found in literature for plasticized PLA (Burgos et al. 2013; Martino et al. 2009) and plasticized PLA_PHB systems 
(Arrieta et al. 2014a; Arrieta et al. 2014b). They are still lower than those obtained for low-density polyethylene, LDPE (around $160 \mathrm{~cm}^{3} \mathrm{~mm} \mathrm{~m}^{-2} \mathrm{day}^{-1}$ ), a polymer currently used in flexible films for food packaging.

As shown in Table 2, no significant $(\mathrm{P}<0.05)$ reduction in the WVP of neat PLA was detected after blending with 15 wt\% of PHB, despite the hydrophobic character provided by PHB to these blends (Arrieta et al. 2014b). The same situation was also observed for PLA_PHB films after the addition of carvacrol (10 wt $\%)$. This unchanged behavior can be explained by the action of some opposite factors that could affect, in a different degree, the barrier properties to water vapor. The increase in the free volume available in the PLA_PHB structure due to the plasticizing effect of carvacrol (Armentano et al. 2015b; Byun et al. 2010; Jamshidian et al. 2012), and the decrease in the hydrophobic character provided by the polar hydroxyl groups of carvacrol (Nostro and Papalia 2012), can be counterbalanced by the high crystallinity and hydrophobic nature of PHB (Arrieta et al. 2014a). The action of all these factors resulted in no overall effect on the WVP of these blends. However, a significant $(\mathrm{P}<0.05)$ increase in the WVP values of PLA_PHB films (around $30 \%$ ) was observed in the quaternary composites with the addition of carvacrol and OLA (15 and 20 wt \%). This result is indicative of the effect of both additives in the barrier to water vapor, in concordance with the OTR*e results already discussed. The increase in free volume for the plasticized PLA_PHB films due to the combined effect of OLA and carvacrol, and the decrease in the hydrophobic character caused by the presence of hydroxyl groups in both additives, resulted in the overall decrease in the resistance of films to water vapor transmission.

\section{Overall Migration}

Overall migration tests provide the total amount of non-volatile substances that could transfer from packaging materials to food and the calculated value should be lower than the limit established in the current legislation $\left(60 \mathrm{mg} \mathrm{kg}^{-1}\right)(\mathrm{EC}$ 2011). But active packaging systems could be considered as the exception to the rule, since the transfer of selected additives to foodstuff could provide beneficial effects. Table 3 shows the overall migration values of neat PLA and PLA_PHB-based formulations in ethanol $10 \%(\mathrm{v} / \mathrm{v})$ and isooctane.

\section{Table 3}

In the case of ethanol $10 \%(\mathrm{v} / \mathrm{v})$ all materials showed overall migration values lower than the current limit (EC 2011). It was observed that PLA_15PHB films showed the highest value, but no significant differences $(\mathrm{P}<0.05)$ were observed when compared to neat PLA. It is important to highlight that polarity and solubility are controlling factors in the migration process of non-volatile compounds, due to the expected interactions between polymer matrices, food simulants and potential migration compounds. Since PLA and PHB are insoluble in water, due to their high non-polar nature, the polar hydroxyl groups of carvacrol decreased the hydrophobic character of the polymer (Nostro and Papalia 2012). Therefore, high affinity of this active compound with ethanol $10 \%$ (v/v) should be expectable (Jamshidian et al. 2012; Zygoura et al. 2011; Suppakul et al. 2011). However, it was observed that the overall migration for PLA_PHB 
films with carvacrol decreased significantly $(\mathrm{P}<0.05)$ in contact with this aqueous simulant. This behavior could be explained by the interactions between hydroxyl groups of carvacrol with both hydrophobic polymers, increasing its retention in the polymer matrix and consequently restricting the release of the active compound into the polar compound used as food simulant (Arrieta et al. 2014a). The presence of OLA in these formulations improved the hydrophobic character of the blend due to its non-polar nature, improving interactions with carvacrol and leading to the reduction in the overall migration resulting in no detection of migration in these films (Armentano et al. 2015a).

On the other hand, migration tests performed in the non-polar simulant (isooctane) showed that PLA_PHB formulations with OLA and carvacrol exceeded the overall migration limit $\left(60 \mathrm{mg} \mathrm{kg}^{-1}\right)$, while the un-plasticized samples showed lower values, accomplishing the current legislation. This behavior could be explained by the plasticizing effect of both additives resulting in the increase of the mobility of low molar mass compounds through the polymer chains and the crystallinity of films, in agreement with the previously reported thermal studies (Armentano et al. 2015a). In addition, PLA, PHB and OLA could show high affinity with isooctane by their non-polar nature. Conversely some improvement in the penetration capacity of isooctane through the PLA_PHB matrix might happen, thereby increasing the release of carvacrol and other low molar mass compounds resulting from polymer chains scission after processing or degradation at high temperatures (Ramos et al. 2014a; Suppakul et al. 2011; Fortunati et al. 2012).

In summary, the higher migration observed for PLA_PHB_OLA_Carv based active systems in fatty simulants can be considered as a positive feature for the protection of foodstuff with high fat content since the active agent could be in contact with food for the whole shelf-life.

\section{Antioxidant Activity}

The antioxidant performance of the PLA_PHB based films containing the active agent, carvacrol, was determined by the scavenging activity of their methanol extracts against the DPPH radical. The obtained results are shown in Table 4.

\section{Table 4}

All extracts containing carvacrol exhibited inhibition of the DPPH radical, with no significant differences $(\mathrm{P}<0.05)$ between samples. This result indicates that the amount of the additive remaining in the polymer matrix after processing (around $7.6 \mathrm{wt} \%$ ) (Armentano et al. 2015a), corresponds to about $0.4 \mathrm{mg} \mathrm{mL}^{-1}$ in the extracts, showing an appreciable antioxidant activity. It has been reported that the antioxidant character of carvacrol is based on the ability of the hydroxyl groups to reduce the DPPH radical by donating hydrogen atoms (Wu et al. 2012; López-Mata et al. 2013). Furthermore, the presence of the phenolic group in the carvacrol molecules can affect their antioxidant ability (Mastelić et al. 2008). Other authors reported similar results (more than $50 \%$ DPPH inhibition) of carvacrol extracts with concentrations between $0.3-1 \mathrm{mg} \mathrm{mL}^{-1}$ in different polymer matrices, such as polypropylene (Ramos et al. 2014a), 
chitosan (López-Mata et al. 2013) and zein (Wu et al. 2012). From these results it can be concluded that carvacrol is

able to act as an efficient antioxidant agent in PLA_PHB and PLA_PHB_OLA based formulations.

\section{Antimicrobial activity}

The antimicrobial performance of the PLA_PHB based films with carvacrol as active agent was firstly evaluated by the agar disk diffusion method to simulate food wrapping, indicating what might happen when the antimicrobial agent migrates from the film to the food in contact with (Erdohan et al. 2013; Guarda et al. 2011). The main results of these antimicrobial tests against two food-borne bacteria, S. aureus (Gram-positive) and E. coli (Gram-negative) are shown in Fig. 1. A remarkable growth of both bacterial cultures on agar plates with PLA, PLA_15PHB and PLA_15PHB_10Car was observed with no significant action of the active agent, while PLA_PHB based films with OLA and carvacrol exhibited some antimicrobial activity against $S$. aureus with the observation of a clear zone of growth inhibition around films after $24 \mathrm{~h}$ (Fig. 1(b)). These results suggest that the addition of OLA to PLA_PHB blends increases mobility of the macromolecular chains promoting the diffusion of carvacrol from the polymer matrix into the agar medium in a radial way, improving the antimicrobial action against $S$. aureus. These results are in agreement with the increase in the migration rate for the quaternary blends, since the plasticizing effect of OLA and carvacrol on PLA_PHB matrices and the increased affinity with fatty mediums, such agar, could explain the high diffusion of carvacrol and the consequent antimicrobial performance of these materials. Nevertheless, the release of the antimicrobial compound from PLA_PHBcarvacrol based films could not be fast enough to produce a visually appreciable inhibition growth in both bacterial cultures, as it was reported by other authors (Guarda et al. 2011; Ramos et al. 2012).

On the other hand, no inhibition halo was observed in the agar plates inoculated with $E$. coli, even for those films with carvacrol and OLA (Fig. 1(a)). This result could be explained by the higher sensitivity of S. aureus (Gram-positive) to carvacrol than in the case of E. coli (Gram-negative) (Guarda et al. 2011; Burt 2004). The explanation of these differences lies on the hydrophobic nature of carvacrol, permitting the insertion of molecules between the fatty acid chains in the lipid bilayer of the cytoplasmic membranes, increasing their fluidity and permeability (Cristani et al. 2007; Lambert et al. 2001; Ultee et al. 1999). This insertion could also change the fatty acid composition of the cytoplasmic membrane, decreasing the cells size (Di Pasqua et al. 2006; La Storia et al. 2011). In the case of Gram-negative bacteria, the presence of carvacrol causes the disruption of their cell membrane and the release of lipo-polysaccharides, resulting in microorganism's death by the increase in the passive permeability of cells as well as the alteration of their electrical equilibrium (Xu et al. 2008). Thus, higher amounts of the antimicrobial agent are required to obtain the same antibacterial effect in Gram-negative bacteria (Burt 2004; Helander et al. 1998). In particular, it was reported that the minimal concentration of carvacrol to inhibit the growth of $E$. coli was $375 \mathrm{ppm}$, while the concentration needed in the case of S. aureus was $225 \mathrm{ppm}$ (Guarda et al. 2011). The results in the present study are in agreement with those 
reported by other authors in active polymer films containing carvacrol or different essential oils with high content of this active compound (López et al. 2007; López-Mata et al. 2013; Guarda et al. 2011; Ramos et al. 2012; Arrieta et al. 2014b; Nostro and Papalia 2012).

Fig. 1

It must be considered that the experimental conditions used in these tests could be not strong enough to obtain the highest potential resistance of specific microorganisms against carvacrol (López et al. 2007). In this sense, further in vitro studies were assessed to get the quantitative antimicrobial potential of the active PLA_PHB films by direct contact of both bacterial suspensions using the $\mathrm{CFU} / \mathrm{mL}$ method.

Fig. 2 shows the viability of $S$. aureus and E. coli cells onto PLA_PHB plasticised films with carvacrol after incubation at $37{ }^{\circ} \mathrm{C}$ for 3 and $24 \mathrm{~h}$, respectively. A difference in viability for both bacterial strains between films with/without carvacrol was observed at both times. In agreement with those results obtained with the agar disk diffusion method, PLA and PLA_PHB films without carvacrol did not show any significant antibacterial activity at both incubation times $(\mathrm{P}<0.05)$ regardless of the tested bacterial strains, but PLA_PHB_10Carv films with/without OLA showed some inhibitory effect against both bacterial strains and incubation times. The observed trend in this activity was PLA_PHB_10Carv < PLA_PHB_20OLA_10Carv < PLA_PHB_15OLA_10Carv. Moreover, the antimicrobial activity against both strains of PLA_PHB films was significantly enhanced by the presence of both 10 wt $\%$ of carvacrol and OLA (15 or $20 \mathrm{wt} \%$ ), showing a bactericidal effect just after $3 \mathrm{~h}$ of incubation that is maintained after $24 \mathrm{~h}$ (Fig. 2A and Fig 2B).

Carvacrol and its essential oils, have been suggested as efficient antimicrobial agents (Nostro and Papalia 2012), but our results showed that the antibacterial activity of films based on PLA_PHB blends was significantly enhanced with the addition of OLA at $15 \mathrm{wt} \%(\mathrm{P}<0.05)$, while it decreased at higher OLA concentrations $(20$ wt $\%)$, with the same quantity of carvacrol. These results confirmed the data already discussed for barrier and migration properties, suggesting that the increase in free volume induced by OLA in PLA_PHB films and the decrease in the hydrophobic character by the presence of hydroxyl groups in both additives resulted in a higher release of the active agent to limit the microorganisms growth.

Fig. 2

\section{Disintegrability}

Disintegrability under composting conditions was evaluated by visual observation of all films at different times. Fig. 3 shows the change in color and increase in opacity in all films after 3 days of incubation, while they exhibited a considerable surface deformation and fractures starting from the $7^{\text {th }}$ days under composting. After 10 days all films were visibly fractured. The color changes could be a signal that the hydrolytic degradation process of the PLA_PHB matrix 
has started facilitated by the low samples thicknesses $(30-40 \mu \mathrm{m})$. The opacity is due to changes in the refractive index which can be attributed to the water absorption and to the formation of low molecular weight compounds produced by the hydrolytic degradation. Moreover, it should be taken into account that the degradation experiments took place at 58 ${ }^{\circ} \mathrm{C}$, which is higher than the $\mathrm{T}_{\mathrm{g}}$ of the polymer matrix. This effect could increase the chain mobility, inducing the crystallization of the PLA matrix and consequently increasing opacity. After 7 days of the test, fragmentation and weight loss were observed for all samples except for the neat PLA and PLA_PHB films, which started fragmentation after 10 days.

The visual observation was confirmed by the disintegrability results (Fig. 4), that remained constant for all systems up to 7 days, while reaching $40 \%$ after 10 days and $70 \%$ after 14 days. The disintegration tests (Figs. 3-4) showed that materials were visibly disintegrated after 17 days.

Fig. 3

The fast appearance of visual signs of degradation in plasticized films and the high percentage of disintegration in systems with OLA and carvacrol after day 7 can be attributed to the low molecular weight and highly mobile chains provided by OLA

Fig.4

\section{Conclusions}

This study shows the potential use of bio-based films based on PLA_PHB blends as one hurdle technology that, in combination with good manufacturing practices and adequate storage temperatures, will permit a significant increase in food quality and shelf-life. The addition of carvacrol improved the antioxidant activity and the antibacterial properties of PLA_15PHB plasticized films. Moreover, all the tested bio-based films disintegrated completely under composting conditions after 17 days, highlighting that the modification of PLA by blending with the highly crystalline PHB did not affect the disintegration properties of the PLA matrix. The use of antimicrobial packaging may contribute to improve the safety in minimally processed foods and consequently the use of PLA_PHB_Carv films could be considered as an interesting alternative to other non-biodegradable materials currently used in food packaging materials.

\section{Acknowledgments}

This work was funded by the SAMSUNG GRO PROGRAMME 2012 and the Spanish Ministry of Economy and Competitiveness (Ref. MAT2014-59242-C2-2-R and MAT2014-55778-REDT).

Conflict of Interest: The authors declare that they have no conflict of interest. 


\section{References}

Alboofetileh, M., Rezaei, M., Hosseini, H., \& Abdollahi, M. (2014). Antimicrobial activity of alginate/clay nanocomposite films enriched with essential oils against three common foodborne pathogens. Food Control, $36(1), 1-7$.

Armentano, I., Fortunati, E., Burgos, N., Dominici, F., Luzi, F., Fiori, S., et al. (2015a). Bio-based PLA_PHB plasticized blend films: Processing and structural characterization. LWT - Food Science and Technology, 64(2), 980-988.

Armentano, I., Fortunati, E., Burgos, N., Dominici, F., Luzi, F., Fiori, S., et al. (2015b). Processing and characterization of plasticized PLA/PHB blends for biodegradable multiphase systems. Express Polymer Letters, 9(7), 583-596.

Arrieta, M. P., Castro-López, M. M., Rayón, E., Barral-Losada, L. F., López-Vilariño, J. M., López, J., et al. (2014a). Plasticized Poly(lactic acid)-Poly(hydroxybutyrate) (PLA-PHB) Blends Incorporated with Catechin Intended for Active Food-Packaging Applications. Journal of Agricultural and Food Chemistry, 62(41), 10170-10180.

Arrieta, M. P., López, J., Hernández, A., \& Rayón, E. (2014a). Ternary PLA-PHB-Limonene blends intended for biodegradable food packaging applications. European Polymer Journal, 50, 255-270.

Arrieta, M. P., Peltzer, M. A., López, J., Garrigós, M. D. C., Valente, A. J. M., \& Jiménez, A. (2014b). Functional properties of sodium and calcium caseinate antimicrobial active films containing carvacrol. Journal of Food Engineering, 121(1), 94-101.

Arrieta, M. P., Samper, M. D., López, J., \& Jiménez, A. (2014b). Combined Effect of Poly(hydroxybutyrate) and Plasticizers on Polylactic acid Properties for Film Intended for Food Packaging. Journal of Polymers and the Environment, 22(4), 460-470.

ASTM (2005). Standard Test Methods for Water Vapor Transmission of Materials. In ASTM E-96/E 96M-05: American Society for Testing and Materials.

Boumail, A., Salmieri, S., Klimas, E., Tawema, P. O., Bouchard, J., \& Lacroix, M. (2013). Characterization of Trilayer Antimicrobial Diffusion Films (ADFs) Based on Methylcellulose-Polycaprolactone Composites. Journal of Agricultural and Food Chemistry, 61(4), 811-821.

Burgos, N., Martino, V. P., \& Jiménez, A. (2013). Characterization and ageing study of poly(lactic acid) films plasticized with oligomeric lactic acid. Polymer Degradation and Stability, 98(2), 651-658.

Burgos, N., Tolaguera, D., Fiori, S., \& Jiménez, A. (2014). Synthesis and Characterization of Lactic Acid Oligomers: Evaluation of Performance as Poly(Lactic Acid) Plasticizers. Journal of Polymers and the Environment, 22(2), 227-235. 
Burt, S. (2004). Essential oils: their antibacterial properties and potential applications in foods—a review. International Journal of Food Microbiology, 94(3), 223-253.

Byun, Y., Kim, Y. T., \& Whiteside, S. (2010). Characterization of an antioxidant polylactic acid (PLA) film prepared with $\alpha$-tocopherol, BHT and polyethylene glycol using film cast extruder. Journal of Food Engineering, $100(2), 239-244$

CLSI (2015). Performance standards for antimicrobial disk susceptibility tests; Approved standard_Twelfth Edition In CLSI document MO2-A12 Wayne, PA: Clinical and Laboratory Standards Institute.

Coma, V. (2008). Bioactive packaging technologies for extended shelf life of meat-based products. Meat Science, 78(12), 90-103.

Cristani, M., D'Arrigo, M., Mandalari, G., Castelli, F., Sarpietro, M. G., Micieli, D., et al. (2007). Interaction of four monoterpenes contained in essential oils with model membranes: Implications for their antibacterial activity. Journal of Agricultural and Food Chemistry, 55(15), 6300-6308.

Chaiwutthinan, P., Pimpan, V., Chuayjuljit, S., \& Leejarkpai, T. (2015). Biodegradable Plastics Prepared from Poly(lactic acid), Poly(butylene succinate) and Microcrystalline Cellulose Extracted from Waste-Cotton Fabric with a Chain Extender. Journal of Polymers and the Environment, 23(1), 114-125.

De Silva, R. T., Pasbakhsh, P., Lee, S. M., \& Kit, A. Y. (2015). ZnO deposited/encapsulated halloysite-poly (lactic acid) (PLA) nanocomposites for high performance packaging films with improved mechanical and antimicrobial properties. Applied Clay Science, 111, 10-20.

Delgado, P. A., \& Hillmyer, M. A. (2014). Combining block copolymers and hydrogen bonding for poly(lactide) toughening. RSC Advances, 4(26), 13266-13273.

Di Pasqua, R., Hoskins, N., Betts, G., \& Mauriello, G. (2006). Changes in Membrane Fatty Acids Composition of Microbial Cells Induced by Addiction of Thymol, Carvacrol, Limonene, Cinnamaldehyde, and Eugenol in the Growing Media. Journal of Agricultural and Food Chemistry, 54(7), 2745-2749.

EC (2002). Commission Directive 2002/72/EC relating to plastic materials and articles intended to come into contact with foodstuffs. In Official Journal of European Communities.

EC (2011). Commission Regulation EU No 10/2011 on plastic materials and articles intended to come into contact with food. In Official Journal of European Communities

Erdohan, Z. Ö., Çam, B., \& Turhan, K. N. (2013). Characterization of antimicrobial polylactic acid based films. Journal of Food Engineering, 119(2), 308-315.

Fiori, S., \& Ara, P. (2009). Method for plasticizing lactic acid polymers. World Patent. 
Fortunati, E., Peltzer, M., Armentano, I., Torre, L., Jiménez, A., \& Kenny, J. M. (2012). Effects of modified cellulose nanocrystals on the barrier and migration properties of PLA nano-biocomposites. Carbohydrate Polymers, 90(2), 948-956.

Guarda, A., Rubilar, J. F., Miltz, J., \& Galotto, M. J. (2011). The antimicrobial activity of microencapsulated thymol and carvacrol. International Journal of Food Microbiology, 146(2), 144-150.

Hakkarainen, M., Karlsson, S., \& Albertsson, A. C. (2000). Rapid (bio)degradation of polylactide by mixed culture of compost microorganisms - low molecular weight products and matrix changes. Polymer, 41(7), 2331-2338.

Helander, I. M., Alakomi, H. L., Latva-Kala, K., Mattila-Sandholm, T., Pol, I., Smid, E. J., et al. (1998). Characterization of the Action of Selected Essential Oil Components on Gram-Negative Bacteria. Journal of Agricultural and Food Chemistry, 46(9), 3590-3595.

Hwang, S. W., Shim, J. K., Selke, S. E. M., Soto-Valdez, H., Matuana, L., Rubino, M., et al. (2012). Poly(L-lactic acid) with added $\alpha$-tocopherol and resveratrol: optical, physical, thermal and mechanical properties. Polymer International, 61(3), 418-425.

ISO (2004). Plastics. Determination of the degree of disintegration of plastic materials under simulated composting conditions in a laboratory-scale test. In ISO 20200:2004: International Organization for Standardization.

Jamshidian, M., Tehrany, E. A., Imran, M., Akhtar, M. J., Cleymand, F., \& Desobry, S. (2012). Structural, mechanical and barrier properties of active PLA-antioxidant films. Journal of Food Engineering, 110(3), 380-389.

Kunioka, M., Ninomiya, F., \& Funabashi, M. (2006). Biodegradation of poly(lactic acid) powders proposed as the reference test materials for the international standard of biodegradation evaluation methods. Polymer Degradation and Stability, 91(9), 1919-1928.

La Storia, A., Ercolini, D., Marinello, F., Di Pasqua, R., Villani, F., \& Mauriello, G. (2011). Atomic force microscopy analysis shows surface structure changes in carvacrol-treated bacterial cells. Research in Microbiology, 162(2), 164-172.

Lambert, R. J. W., Skandamis, P. N., Coote, P. J., \& Nychas, G. J. E. (2001). A study of the minimum inhibitory concentration and mode of action of oregano essential oil, thymol and carvacrol. Journal of Applied Microbiology, 91(3), 453-462.

López-Mata, M. A., Ruiz-Cruz, S., Silva-Beltrán, N. P., Ornelas-Paz, J. D. J., Zamudio-Flores, P. B., \& Burruel-Ibarra, S. E. (2013). Physicochemical, antimicrobial and antioxidant properties of chitosan films incorporated with carvacrol. Molecules, 18(11), 13735-13753.

López, P., Sánchez, C., Batlle, R., \& Nerín, C. (2007). Development of flexible antimicrobial films using essential oils as active agents. Journal of Agricultural and Food Chemistry, 55(21), 8814-8824. 
Martino, V. P., Jiménez, A., \& Ruseckaite, R. A. (2009). Processing and characterization of poly(lactic acid) films plasticized with commercial adipates. Journal of Applied Polymer Science, 112(4), 2010-2018.

Mastelić, J., Jerković, I., Blažević, I., Poljak-Blaži, M., Borović, S., Ivančić-Baće, I., et al. (2008). Comparative Study on the Antioxidant and Biological Activities of Carvacrol, Thymol, and Eugenol Derivatives. Journal of Agricultural and Food Chemistry, 56(11), 3989-3996.

Nostro, A., \& Papalia, T. (2012). Antimicrobial activity of carvacrol: Current progress and future prospectives. Recent Patents on Anti-Infective Drug Discovery, 7(1), 28-35.

Ramos, M., Beltrán, A., Peltzer, M., Valente, A. J. M., \& Garrigós, M. d. C. (2014a). Release and antioxidant activity of carvacrol and thymol from polypropylene active packaging films. LWT - Food Science and Technology, 58(2), $470-477$.

Ramos, M., Jiménez, A., Peltzer, M., \& Garrigós, M. C. (2012). Characterization and antimicrobial activity studies of polypropylene films with carvacrol and thymol for active packaging. Journal of Food Engineering, 109(3), 513-519.

Ramos, M., Jiménez, A., Peltzer, M., \& Garrigós, M. C. (2014b). Development of novel nano-biocomposite antioxidant films based on poly (lactic acid) and thymol for active packaging. Food Chemistry, 162, 149-155.

Salmieri, S., Islam, F., Khan, R. A., Hossain, F. M., Ibrahim, H. M. M., Miao, C., et al. (2014). Antimicrobial nanocomposite films made of poly(lactic acid)-cellulose nanocrystals (PLA-CNC) in food applications: part A - effect of nisin release on the inactivation of Listeria monocytogenes in ham. Cellulose, 21(3), 1837-1850.

Sanchez-Garcia, M. D., Ocio, M. J., Gimenez, E., \& Lagaron, J. M. (2008). Novel Polycaprolactone Nanocomposites Containing Thymol of Interest in Antimicrobial Film and Coating Applications. Journal of Plastic Film and Sheeting, 24(3-4), 239-251.

Suppakul, P., Sonneveld, K., Bigger, S. W., \& Miltz, J. (2011). Diffusion of linalool and methylchavicol from polyethylene-based antimicrobial packaging films. LWT - Food Science and Technology, 44(9), 1888-1893.

Ultee, A., Kets, E. P. W., \& Smid, E. J. (1999). Mechanisms of action of carvacrol on the food-borne pathogen. Applied and Environmental Microbiology, 65(10), 4606-4610.

Wu, Y., Luo, Y., \& Wang, Q. (2012). Antioxidant and antimicrobial properties of essential oils encapsulated in zein nanoparticles prepared by liquid-liquid dispersion method. LWT - Food Science and Technology, 48(2), 283290.

Xu, J., Zhou, F., Ji, B. P., Pei, R. S., \& Xu, N. (2008). The antibacterial mechanism of carvacrol and thymol against Escherichia coli. Letters in Applied Microbiology, 47(3), 174-179. 
Zhang, M., \& Thomas, N. L. (2011). Blending polylactic acid with polyhydroxybutyrate: The effect on thermal,

1 mechanical, and biodegradation properties. Advances in Polymer Technology, 30(2), 67-79.

Zygoura, P. D., Paleologos, E. K., \& Kontominas, M. G. (2011). Changes in the specific migration characteristics of packaging-food simulant combinations caused by ionizing radiation: Effect of food simulant. Radiation Physics and Chemistry, 80(8), 902-910. 


\section{Figure Captions}

Fig. 1 Antimicrobial activity of neat PLA and PLA_PHB-based films against (a) E. coli and (b) S. aureus.

Fig. 2 Antibacterial properties of PLA_PHB plasticized blend films. S. aureus and E.coli cells were incubated on PLA_PHB plasticized blend films with /without carvacrol $10 \mathrm{wt} \%$ for $3 \mathrm{~h}(\mathrm{~A})$ and $24 \mathrm{~h}(\mathrm{~B})$ at $37{ }^{\circ} \mathrm{C}$, respectively. Results are expressed as a percent of bacterial cells grown on PLA film and set equal to $100 \%$. Data are presented as the average of three replicates \pm standard deviation.

Fig. 3 Visual observation of PLA and PLA_PHB films at different stages of incubation in composting conditions.

Fig. 4 Disintegrability percentage values of PLA and PLA_PHB systems at different stages of incubation in composting conditions. The line at $90 \%$ represents the goal of disintegrability test as reported in the ISO 20200. 
(a) E.coli
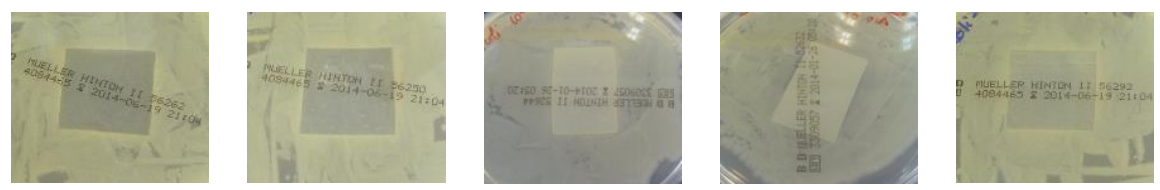

(b) S.aureus
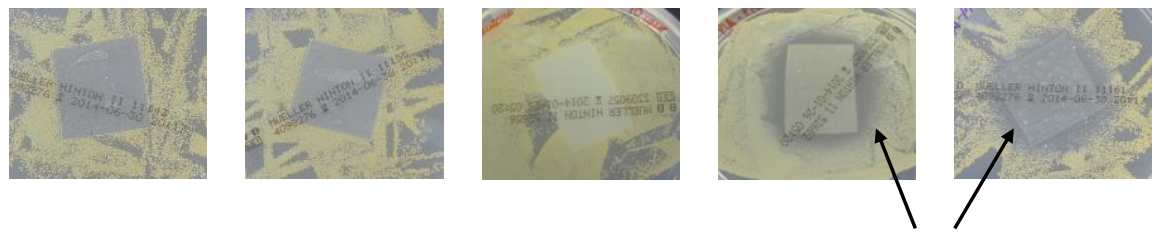

Inhibition zone 


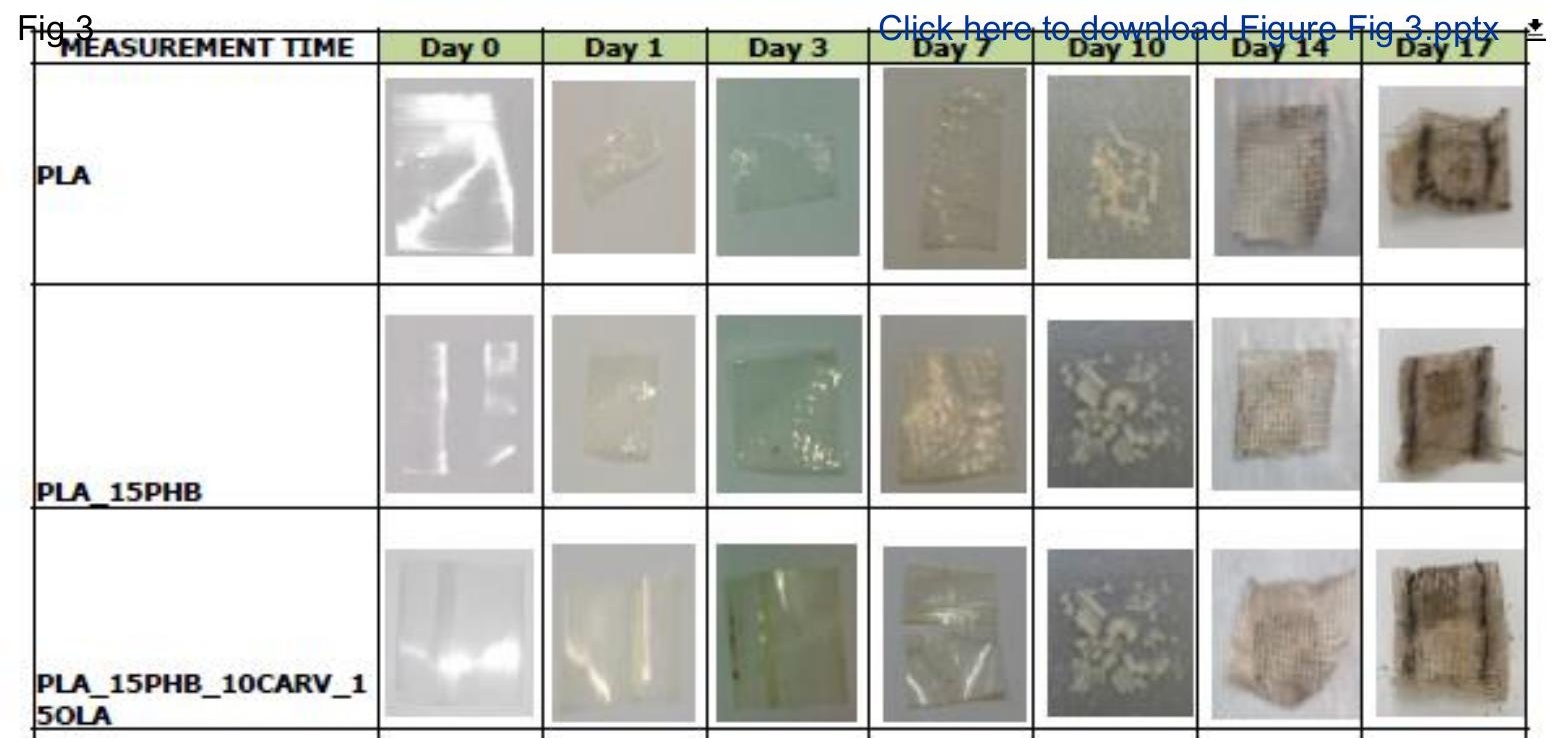




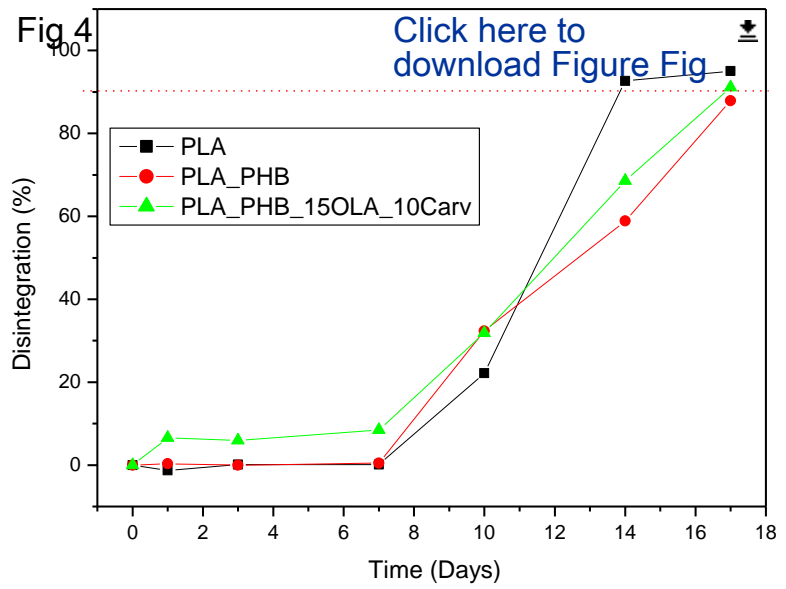


Table 1. Material formulations.

\begin{tabular}{|l|c|c|c|c|}
\hline \multirow{2}{*}{ Formulations } & \multicolumn{4}{|c|}{ Component contents (wt\%) } \\
\cline { 2 - 5 } & PLA & PHB & Carvacrol & OLA \\
\hline PLA & 100 & 0 & 0 & 0 \\
\hline PLA_15PHB & 85 & 15 & 0 & 0 \\
\hline PLA_15PHB_10Carv & 75 & 15 & 10 & 0 \\
\hline PLA_15PHB_15OLA_10Carv & 60 & 15 & 10 & 15 \\
\hline PLA_15PHB_20OLA_10Carv & 55 & 15 & 10 & 20 \\
\hline
\end{tabular}


Table 2. Oxygen Transmission rate per film thickness (OTR*e) and Water Vapor Permeability (WVP) coefficients for PLA_PHB based films.

\begin{tabular}{|c|c|c|}
\hline Formulations & $\begin{array}{c}\text { OTR } * \mathrm{e} \\
\left(\mathrm{cm}^{3} \cdot \mathrm{mm}^{*} \mathbf{m}^{-2} \cdot \mathrm{day}^{-1}\right)\end{array}$ & 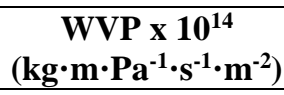 \\
\hline$P L A$ & $22.9 \pm 0.4^{\mathrm{a}}$ & $1.88 \pm 0.21 \mathrm{ab}$ \\
\hline PLA_15PHB & $14.9 \pm 0.8^{b}$ & $1.54 \pm 0.20^{\mathrm{ac}}$ \\
\hline$P L A \_15 P H B \_10$ Carv & $20.7 \pm 3.3^{a}$ & $1.41 \pm 0.19^{c}$ \\
\hline PLA_15PHB_15OLA_10 Carv & $63.3 \pm 2.8^{c}$ & $2.03 \pm 0.11^{b}$ \\
\hline PLA_15PHB_20OLA_10 Carv & $76.0 \pm 2.7^{\mathrm{d}}$ & $1.95 \pm 0.28^{b}$ \\
\hline
\end{tabular}

${ }^{\text {a-d }}$ Different superscripts within the same column indicate significant differences between formulations $(\mathrm{P}<0.05)$ $\mathrm{n}=3$, mean $\pm \mathrm{SD}$. 
Table 3. Overall migration values $\left(\mathrm{mg} \mathrm{kg}^{-1}\right)$ in ethanol $10 \%(\mathrm{v} / \mathrm{v})$ and isooctane for PLA_PHB based films.

\begin{tabular}{|l|c|c|}
\hline Formulations & $\begin{array}{c}\text { Ethanol 10 \% (v/v) } \\
\left(\mathrm{mg} \mathrm{kg}^{-1}\right)\end{array}$ & $\begin{array}{c}\text { Isooctane } \\
\left(\mathrm{mg} \mathrm{kg}^{-1}\right)\end{array}$ \\
\hline$P L A$ & $19 \pm 2^{\mathrm{ab}}$ & $16 \pm 3^{\mathrm{a}}$ \\
\hline PLA_15PHB & $27 \pm 9^{\mathrm{b}}$ & n.d. \\
\hline PLA_15PHB_10Carv & $16 \pm 1^{\mathrm{a}}$ & $8 \pm 3^{\mathrm{a}}$ \\
\hline PLA_15PHB_15OLA_10Carv & n.d. & $250 \pm 16^{\mathrm{b}}$ \\
\hline PLA_15PHB_20OLA_10Carv & n.d. & $255 \pm 23^{\mathrm{b}}$ \\
\hline
\end{tabular}

${ }^{\mathrm{a}-\mathrm{b}}$ Different superscripts within the same column indicate significant differences between formulations $(\mathrm{P}<0.05)$ n.d.: not detected, $\mathrm{n}=3$, mean $\pm \mathrm{SD}$. 
Table 4. Radical scavenging activity obtained by the DPPH method, expressed as percent of inhibition, for the formulations containing carvacrol.

\begin{tabular}{|l|c|}
\hline Formulations & Inhibition (\%) \\
\hline PLA_15PHB_10Carv & $64 \pm 2^{\mathrm{a}}$ \\
\hline PLA_15PHB_15OLA_10Carv & $56 \pm 6^{\mathrm{a}}$ \\
\hline PLA_15PHB_20OLA_10Carv & $58 \pm 4^{\mathrm{a}}$ \\
\hline
\end{tabular}

${ }^{a}$ Different superscripts within the same column indicate significant differences between formulations $(\mathrm{P}<0.05)$ $\mathrm{n}=3$, mean $\pm \mathrm{SD}$. 